\title{
Stabilization Criteria for Bilinear Systems with Time-varying Delay
}

\author{
Pin-Lin Liu \\ Department of Automation Engineering Institute of Mechatronoptic System, Chienkuo Technology University, Taiwan
}

Copyright @ 2014 Horizon Research Publishing All rights reserved.

\begin{abstract}
In this paper we consider the problem of stabilizing a bilinear system with time- varying delay via linear state feedback control. Based on the Lyapunov method, a delay-dependent criterion for determining the stabilization of system is obtained in terms of linear matrix inequalities (LMIs) and used to express the relationships between the terms in the Leibniz-Newton formula, which can be easily solved by efficient convex optimization algorithms. From the numerical examples, the obtained results have some significant improvements over the recent literatures.
\end{abstract}

Keywords Bilinear system, time-varying delay, linear matrix inequalities (LMIs), convex optimization algorithms.

\section{Introduction}

The phenomena of time-delay are very often encountered in control systems, economic systems and even population dynamics, etc. The presence of time-delay in the control or state evolution laws usually degrades system performance or even causes instability. Since delay is usually time-varying in many practical systems, many approaches have been developed to derive the delay-dependent stability criteria for systems with time-varying delays. Thus, the problem of stability analysis for time-delay systems has been an important topic [1-12] and references therein. The stability criteria for linear system with delayed state can be classified in two classes function on their dependence on the size of delay: delay-independent [7] or delay-dependent [2-6, 8-12]. For systems with time-varying delays, the above-cited literature usually demand that the upper bound of the derivative of delays must be smaller than 1 . This implies that information on the derivative of time-delay term $h(t)$ is not used, which is obviously unreasonable. If the upper bound of derivative of delays is larger than 1, results in [2, 3, 4, 5, 7, 9-12] do not apply. Recently, using various analyzing methods, the upper bounds of delay derivative larger than or equal to 1 have been presented [6]. In order to reduce the conservativeness of the stability and stabilization criteria, many methods have been proposed, such as the model transformation method [2], augmented Lyapunov- Krasovskii functional method [10], free weighting matrices method [3], Jensen's inequality [6] and delay-partitioning method [8].

On the other hand, bilinear systems have found a rich field of applications in many areas of signal process and control theory. Bilinear systems are derived from basic phenomena as natural models to describe the dynamics of numerous processes in physics, biochemistry, and agriculture, as well as engineering. Furthermore, when dealing with nonlinear systems it is often useful to consider bilinearization as opposed to linearization, in order to obtain a mathematical model representation that is applicable for a wider range about a given operating point [7]. The great interest on the bilinear systems is due to their simplicity and capability to describe real processes. The bilinear mathematical models are always used to model real-world dynamic systems $[4,5,7,11,12]$. As for the stabilization of bilinear systems with time varying, only a few works have been done on this subject.

More recently, the state feedback control of bilinear systems with time delay has been addressed by means of a linear matrix inequality (LMI)optimization problem in [11, 12], where an estimate of the domain of attraction is also computed. Hence, the stabilization problem of bilinear system is an important task for the control system engineers. However, the result in [11] gives only stability conditions for the closed-loop bilinear time-delay systems with given controllers, and their given controllers are state feedback controllers. It did not propose even state feedback controller design method. Then, the research has extended to an output feedback control design of bilinear time-delay systems in [12] where the state feedback control and observer design have been proposed. As for the stabilization of bilinear systems with time varying, only a few works have been done on this subject. When time-varying delay appears in bilinear systems, the stabilization problem 
becomes more complex and challenging. However, to the best of our knowledge, the problem of stabilization for bilinear systems with time-varying delay remains open, which motivates this paper.

The objective of this paper is to study the problem of stabilization of bilinear systems with time-varying delay by means of state feedback control law. The main results of consist in investigating the conditions dependent on the size of delay for closed-loop stability such a class of systems. All the results are expressed in term of LMIs, which can be easily solved numerically by employing the convex optimization problem algorithm. The advantage of the approach is illustrated by numerical examples.

\section{Stability Description and Preliminaries}

Consider the following bilinear system with time varying delay described by:

$$
\begin{aligned}
& \dot{x}(t)=A_{0} x(t)+A_{1} x(t-h(t))+N_{0} u(t) x(t)+N_{1} u(t) x(t-h(t))+B u(t) \\
& x(t)=\phi(t), \quad t \in[-h, 0]
\end{aligned}
$$

where $x(t) \in R^{n}$ is the state vector; $u(t) \in R^{m}$ is the control input vector; is the measurement output; $x_{t}$ is the state at time $t$ denoted by $x_{t}(s):=x(t+s) ; A_{0}, A_{1}, N_{0}, N_{1}$, and $B$ are known constant matrices with appropriate dimensions; $\varphi(t)$ is a smooth vector-valued initial function.

The time-delay $h(t)$ may be unknown but is assumed to be bounded. It is also assumed to be a smooth function such that

$$
0 \leq h(t) \leq h \quad \text { and } \quad \dot{h}(t) \leq h_{d}
$$

where $h$ and $h_{d}$ are some positive constants.

The following lemmas are useful in deriving the criteria.

Lemma 1 [6]: If there exist symmetric positive-definite matrix

$$
X=\left[\begin{array}{ccc}
X_{11} & X_{12} & X_{13} \\
X_{12}^{T} & X_{22} & X_{23} \\
X_{13}^{T} & X_{23}^{T} & X_{33}
\end{array}\right] \geq 0
$$

the following integral inequality holds

$$
\begin{aligned}
& -\int_{t-h(t)}^{t} \dot{x}^{T}(s) X_{33} \dot{x}(s) d s \\
& \leq \int_{t-h(t)}^{t}\left[\begin{array}{lll}
x^{T}(t) & x^{T}(t-h(t)) & \dot{x}^{T}(s)
\end{array}\right]\left[\begin{array}{ccc}
X_{11} & X_{12} & X_{13} \\
X_{12}^{T} & X_{22} & X_{23} \\
X_{13}^{T} & X_{23}^{T} & 0
\end{array}\right]\left[\begin{array}{c}
x(t) \\
x(t-h(t)) \\
\dot{x}(s)
\end{array}\right] d s
\end{aligned}
$$

Lemma 2[1]: The following matrix inequality

$$
\left[\begin{array}{ll}
Q(x) & S(x) \\
S^{T}(x) & R(x)
\end{array}\right]<0
$$

where $Q(x)=Q^{T}(x), R(x)=R^{T}(x)$ and $S(x)$ depend on affine on $x$, is equivalent to

$$
\begin{aligned}
& R(x)<0, \\
& Q(x)<0,
\end{aligned}
$$

and

$$
Q(x)-S(x) R^{-1}(x) S^{T}(x)<0 .
$$

\section{State Feedback Control for Time-Varying Delay Systems}

In the section, we will focus on the controller synthesis for the bilinear system with time-varying delay (1) and the objective is to construct a state-feedback control law. 


$$
u(t)=\frac{\rho K x(t)}{\sqrt{1+x^{T}(t) K^{T} K x(t)}}
$$

where $\rho$ is a given scalar, and $K$ is a constant matrix to be determined. Then, the natural choice of a controller is given by

$$
\begin{aligned}
& u(t)=\rho \sin \theta(t) \\
& =\rho K \cos \theta(t) x(t)
\end{aligned}
$$

where $\sin \theta(t)=\frac{K x(t)}{\sqrt{1+x^{T}(t) K^{T} K x(t)}}, \cos \theta(t)=\frac{1}{\sqrt{1+x^{T}(t) K^{T} K x(t)}}$, and $\theta \in\left[-\frac{\pi}{2}, \frac{\pi}{2}\right]$.

The closed-loop system (1) with (6) is given by

$$
\dot{x}(t)=\left(A_{0}+\rho B K \cos \theta(t)+N_{0} \rho \sin \theta(t)\right) x(t)+\left(A_{1}+N_{1} \rho \sin \theta(t)\right) x(t-h(t))
$$

A problem is to find a state feedback controller (5) that stabilizes the closed-loop system (5). The form of the controller (5) is bounded by $\rho$, which makes the system

analysis and controller design feasible.

We first give delay-dependent stability conditions of the closed-loop system (7), and then we propose a design method of stabilizing controller for the system (1).

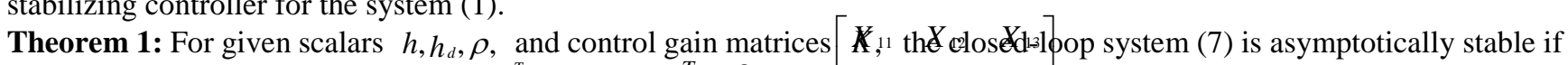
there exist matrices $P=P^{T}>0, \quad Q=Q^{T}>0, R=R^{T}>0, X=\left|\begin{array}{lll}X_{12}^{T} & X_{22} & X_{23}\end{array}\right| \geq 0$, such that the following LMIs hold:

$$
\Omega=\left[\begin{array}{ccccc}
\Omega_{11} & \Omega_{12} & \Omega_{13}^{X_{1}^{1}} & X_{23}^{T} & X_{33} \\
\Omega_{12}^{T} & \Omega_{22} & 0 \\
\Omega_{13}^{T} & 0 & \Omega_{33}
\end{array}\right]<0
$$

and

$$
R-X_{33} \geq 0
$$

where

$$
\begin{aligned}
& \Omega_{11}=A_{0}^{T} P+P A_{0}+N_{0}^{T} N_{0}+N_{1}^{T} N_{1}+K^{T} B^{T} B K+\rho^{2} P^{2}+Q+h X_{11}+X_{13}+X_{13}^{T}+4 h \rho^{2} N_{0}^{T} R N_{0}+5 h A_{0}^{T} R A_{0}, \\
& \Omega_{12}=P A_{1}+h X_{12}-X_{13}+X_{23}^{T}, \\
& \Omega_{13}=\sqrt{3} \rho h K^{T} B^{T} R, \\
& \Omega_{22}=h X_{22}-X_{23}-X_{23}^{T}-\left(1-h_{d}\right) Q+\rho^{2} P+4 h A_{1}^{T} R A_{1}+4 h \rho^{2} N_{1}^{T} R N_{1}, \\
& \Omega_{33}=-h R .
\end{aligned}
$$

Proof: We consider the following Lyapunov-Krasovskii functional candidate:

$$
V\left(x_{t}\right)=V_{1}\left(x_{t}\right)+V_{2}\left(x_{t}\right)+V_{3}\left(x_{t}\right) \text {, }
$$

where

$$
\begin{aligned}
& V_{1}\left(x_{t}\right)=x^{T}(t) P x(t), \\
& V_{2}\left(x_{t}\right)=\int_{t-h(t)}^{t} x^{T}(s) Q x(s) d s, \\
& V_{3}\left(x_{t}\right)=\int_{-h}^{0} \int_{t+\theta}^{t} \dot{x}^{T}(s) R \dot{x}(s) d s d \theta,
\end{aligned}
$$

with matrices $P=P^{T}>0, Q=Q^{T}>0, R=R^{T}>0$, to be determined.

Now, let us calculate the time derivative of $V(t)$ for $t \in[0, \infty)$ along trajectory (7).

$$
\dot{V}\left(x_{t}\right)=\dot{V}_{1}\left(x_{t}\right)+\dot{V}_{2}\left(x_{t}\right)+\dot{V}_{3}\left(x_{t}\right)
$$

First the derivative of $V_{1}(t)$ is 


$$
\begin{aligned}
& \dot{V}_{1}\left(x_{t}\right)=x^{T}(t) P \dot{x}(t)+\dot{x}^{T}(t) P x(t) \\
& =x^{T}(t) P\left[\left(A_{0}+\rho B K \cos \theta(t)+N_{0} \rho \sin \theta(t)\right) x(t)+\left(A_{1}+N_{1} \rho \sin \theta(t)\right) x(t-h(t))\right] \\
& +\left[\left(A_{0}+\rho B K \cos \theta(t)+N_{0} \rho \sin \theta(t)\right) x(t)+\left(A_{1}+N_{1} \rho \sin \theta(t)\right) x(t-h(t))\right]^{T} P x(t) \\
& \left.=x^{T}(t) P\left[A_{0}+\rho B K \cos \theta(t)+N_{0} \rho \sin \theta(t)\right] x(t)+x^{T}(t)\left[A_{0}+\rho B K \cos \theta(t)+N_{0} \rho \sin \theta(t)\right)\right]^{T} P x(t) \\
& +x^{T}(t) P\left(A_{1}+N_{1} \rho \sin \theta(t)\right) x(t-h(t))+x^{T}(t-h(t))\left[\left(A_{1}+N_{1} \rho \sin \theta(t)\right) x(t-h(t))\right]^{T} P x(t) \\
& \leq x^{T}(t)\left[A_{0}^{T} P+P A_{0}+N_{0}^{T} N_{0}+K^{T} B^{T} B K+\rho^{2} P^{2}\left(\sin ^{2} \theta(t)+\cos ^{2} \theta(t)\right)\right] x(t) \\
& +x^{T}(t) P A_{1} x(t-h(t))+x^{T}(t-h(t)) A_{1}^{T} P x(t)+x^{T}(t) N_{1}^{T} N_{1} x(t)+x^{T}(t-h(t)) \rho^{2} P^{2} \sin ^{2} \theta(t) x(t-h(t)) \\
& \leq x^{T}(t)\left[A_{0}^{T} P+P A_{0}+N_{0}^{T} N_{0}+K^{T} B^{T} B K+\rho^{2} P^{2}\right] x(t)+x^{T}(t) P A_{1} x(t-h(t))+x^{T}(t-h(t)) A_{1}^{T} P x(t) \\
& +x^{T}(t) N_{1}^{T} N_{1} x(t)+x^{T}(t-h(t)) \rho^{2} P^{2} x(t-h(t))
\end{aligned}
$$

Second, we get the bound of $V_{2}(t)$ as

$$
\begin{aligned}
& \dot{V}_{2}\left(x_{t}\right)=x^{T}(t) Q x(t)-x^{T}(t-h(t))(1-\dot{h}(t)) Q x(t-h(t)) \\
& \leq x^{T}(t) Q x(t)-x^{T}(t-h(t))\left(1-h_{d}\right) Q x(t-h(t))
\end{aligned}
$$

Third, the bound of $V_{3}(t)$ is as follows:

$$
\begin{aligned}
& \dot{V}_{3}\left(x_{t}\right)=\dot{x}^{T}(t) h R \dot{x}(t)-\int_{t-h}^{t} \dot{x}^{T}(s) R \dot{x}(s) d s \\
& \leq \dot{x}^{T}(t) h R \dot{x}(t)-\int_{t-h(t)}^{t} \dot{x}^{T}(s) R \dot{x}(s) d s \\
& =\dot{x}^{T}(t) h R \dot{x}(t)-\int_{t-h(t)}^{t} \dot{x}^{T}(s)\left(R-X_{33}\right) \dot{x}(s) d s-\int_{t-h(t)}^{t} \dot{x}^{T}(s) X_{33} \dot{x}(s) d s
\end{aligned}
$$

Using Leibniz-Newton formula $x(t)-x\left(t-h(t)=\int_{t-h(t)}^{t} \dot{x}(s) d s\right.$, with Lemma 1 obtains

$$
\begin{aligned}
& -\int_{t-h(t)}^{t} \dot{x}^{T}(s) X_{33} \dot{x}(s) d s \\
& \leq \int_{t-h(t)}^{t}\left[x^{T}(t) \quad x^{T}(t-h(t)) \quad \dot{X}^{T}(s)\right] \\
& {\left[\begin{array}{ccc}
X_{11} & X_{12} & X_{13} \\
X_{12}^{T} & X_{22} & X_{23} \\
X_{13}^{T} & X_{23}^{T} & 0
\end{array}\right]\left[\begin{array}{c}
x(t) \\
x(t-h(t)) \\
\dot{x}(s)
\end{array}\right] d s} \\
& =x^{T}(t)\left[h X_{11}+X_{13}+X_{13}^{T}\right] x(t) \\
& +x^{T}(t)\left[h X_{12}-X_{13}+X_{23}^{T}\right] x(t-h(t)) \\
& +X^{T}(t-h(t))\left[h X_{12}^{T}-X_{13}^{T}+X_{23}\right] X(t) \\
& +X^{T}(t-h)\left[h X_{22}-X_{23}-X_{23}^{T}\right] x(t-h(t))
\end{aligned}
$$

The operator for term $\dot{x}^{T}(t) h R \dot{x}(t)$ is as follows:

$$
\begin{aligned}
& \dot{x}^{T}(t) h R \dot{x}(t) \\
& =\left[\left(A_{0}+\rho B K \cos \theta(t)+N_{0} \rho \sin \theta(t)\right) x(t)+\left(A_{1}+N_{1} \rho \sin \theta(t)\right) x(t-h(t))\right]^{T} \times h R \times \\
& {\left[\left(A_{0}+\rho B K \cos \theta(t)+N_{0} \rho \sin \theta(t)\right) x(t)+\left(A_{1}+N_{1} \rho \sin \theta(t)\right) x(t-h(t))\right]} \\
& =x^{T}(t) h\left(A_{0}+\rho B K \cos \theta(t)+N_{0} \rho \sin \theta(t)\right)^{T} R\left(A_{0}+\rho B K \cos \theta(t)+N_{0} \rho \sin \theta(t)\right) x(t) \\
& +x^{T}(t) h\left(A_{0}+\rho B K \cos \theta(t)+N_{0} \rho \sin \theta(t)\right)^{T} R\left(A_{1}+N_{1} \rho \sin \theta(t)\right) x(t-h(t)) \\
& +x^{T}(t-h(t)) h\left(A_{1}+N_{1} \rho \sin \theta\right)^{T} R\left(A_{0}+\rho B K \cos \theta(t)+N_{0} \rho \sin \theta(t)\right) x(t) \\
& +x^{T}(t-h(t)) h\left(A_{1}+N_{1} \rho \sin \theta(t)\right)^{T} R\left(A_{1}+N_{1} \rho \sin \theta(t)\right) x(t-h(t)) \\
& \leq x^{T}(t) h\left(3 A_{0}^{T} R A_{0}+\rho^{2} N_{0}^{T} R N_{0} \sin ^{2} \theta(t)+\rho^{2} K^{T} B^{T} R B \cos ^{2} \theta(t)+\rho^{2} N_{0}^{T} R N_{0}+\rho^{2} K^{T} B^{T} R B K\right) x(t) \\
& +x^{T}(t) h\left(2 A_{0}^{T} R A_{0}+\rho^{2} N_{0}^{T} R N_{0}+\rho^{2} K^{T} B^{T} R B K+\rho^{2} K^{T} B^{T} R B \sin ^{2} \theta(t)+\rho^{2} N_{0}^{T} R N_{0} \sin ^{2} \theta(t)\right) x(t) \\
& +x^{T}(t-h(t)) h\left(2 A_{1}^{T} R A_{1}+\rho^{2} N_{1}^{T} R N_{1}+\rho^{2} N_{1}^{T} R N_{1} \sin ^{2} \theta(t)\right) x(t-h(t)) \\
& +x^{T}(t-h(t)) h\left(2 A_{1}^{T} R A_{1}+2 \rho^{2} N_{1}^{T} R N_{1} \sin ^{2} \theta(t)\right) x(t-h(t)) \\
& \leq x^{T}(t) h\left(5 A_{0}^{T} R A_{0}+4 \rho^{2} N_{0}^{T} R N_{0}+3 \rho^{2} K^{T} B^{T} R B K\right) x(t)+x^{T}(t-h(t)) h\left(4 A_{1}^{T} R A_{1}+4 \rho^{2} N_{1}^{T} R N_{1}\right) x(t-h(t)),
\end{aligned}
$$




$$
\dot{V}(t) \leq \xi^{T}(t) \Xi \xi(t)-\int_{t-h(t)}^{t} \dot{x}^{T}(s)\left(R-X_{33}\right) \dot{x}(s) d s
$$

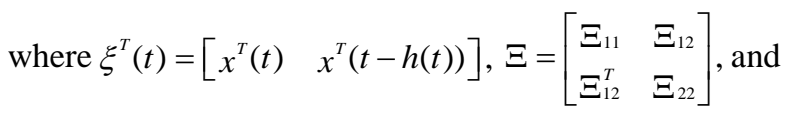

$$
\begin{aligned}
& \Xi_{11}=A_{0}^{T} P+P A_{0}+N_{0}^{T} N_{0}+N_{1}^{T} N_{1}+K^{T} B^{T} B K+\rho^{2} P^{2}+Q+h X_{11}+X_{13}+X_{13}^{T}+3 h \rho^{2} K^{T} B^{T} R B K+4 h \rho^{2} N_{0}^{T} R N_{0}+5 h A_{0}^{T} R A_{0}, \\
& \Xi_{12}=P A_{1}+h X_{12}-X_{13}+X_{23}^{T}, \\
& \Xi_{22}=h X_{22}-X_{23}-X_{23}^{T}-\left(1-h_{d}\right) Q+\rho^{2} P^{2}+4 h A_{1}^{T} R A_{1}+4 h \rho^{2} N_{1}^{T} R N_{1} .
\end{aligned}
$$

From Equation (16) and the Schur complement of Lemma 2, it is easy to see that $\dot{V}\left(x_{t}\right)<0$ holds if $R-X_{33} \geq 0$. If LMIs (8) are feasible, system (7) is asymptotically stable. This completes the proof.

The following Theorem 2 gives an LMI-based computational procedure to determine state-feedback controller. Then we have the following result.

Theorem 2: For given scalars $h, \rho, h_{d}$, the closed-loop system (7) is asymptotically stable if there exist positive-definite matrices $W=W^{T}>0, \quad U=U^{T}>0, \quad V=V^{T}>0, \quad S=S^{T}>0, \quad \bar{X}=\left[\begin{array}{lll}\bar{X}_{11} & \bar{X}_{12} & \bar{X}_{13} \\ \bar{X}_{12}^{T} & \bar{X}_{22} & \bar{X}_{23} \\ \bar{X}_{13}^{T} & \bar{X}_{23}^{T} & \bar{X}_{33}\end{array}\right] \geq 0, \quad$ and any matrix $Y$ with appropriate dimension such that the following LMIs holds:

$$
\Psi=\left[\begin{array}{ccc}
\Psi_{11} & \Psi_{12} & \Psi_{13} \\
\Psi_{12}^{T} & \Psi_{22} & 0 \\
\Psi_{13}^{T} & 0 & \Psi_{33}
\end{array}\right]<0
$$

and

$$
W-\bar{X}_{33} \geq 0
$$

where

$$
\begin{aligned}
& \Psi_{11}=W A_{0}^{T}+A_{0} W+W N_{0}^{T} N_{0} W+W N_{1}^{T} N_{1} W+Y^{T} B^{T} B Y+\rho^{2} I+U+h \bar{X}_{11}+\bar{X}_{13}+\bar{X}_{13}^{T}+4 h \rho^{2} N_{0}^{T} V N_{0}+5 h A_{0}^{T} V A_{0}, \\
& \Psi_{12}=A_{1} W+h \bar{X}_{12}-\bar{X}_{13}+\bar{X}_{23}^{T}, \\
& \Psi_{14}=\sqrt{3} h \rho Y^{T} B^{T}, \\
& \Psi_{22}=h \bar{X}_{22}-\bar{X}_{23}-\bar{X}_{23}^{T}-\left(1-h_{d}\right) U+\rho^{2} I+4 h A_{1}^{T} V A_{1}+4 h \rho^{2} N_{1}^{T} V N_{1}, \\
& \Psi_{33}=-h S .
\end{aligned}
$$

Then, the bilinear system with time-varying delay (7) is asymptotically stable within allowable time delay $h$ under the state feedback control law and $K=Y_{W}{ }^{-1}$ is a stabilizing gain.

Proof: Pre- and post- multiplying both sides of (8) by $\operatorname{diag}\left\{P^{-1}, P^{-1}, R^{-1}\right\}$ and letting $W=P^{-1}, Y=K W$, $P^{-1} Q P^{-1}=U, P^{-1} R P^{-1}=V, P^{-1} X_{i j} P^{-1}=\bar{X}_{i j}(i, j=1,2,3), \quad S=R^{-1}$ and $\left[\begin{array}{cc}R^{-1} & P^{-1}\end{array}\right]\left[\begin{array}{c}R \\ -X_{33}\end{array}\right] P^{-1}=W-\bar{X}_{33}$ leads to (17). This ends the proof.

Remark 1: In the stability problem, maximum allowable delay bound (MADB) $h$ which ensures that bilinear system with time varying delay (1) is stabilizable for any $h$ can be determined by solving the following quasi-convex optimization problem when the other bound of time-varying delay $h$ is known.

$$
\left\{\begin{array}{l}
\text { Maximize } \\
\text { Subject to }
\end{array}\right.
$$

Inequality (18) is a convex optimization problem and can be obtained efficiently using the MATLAB LMI Toolbox.

\section{Illustrative Examples}

This section provides two numerical examples to demonstrate the effectiveness of the presented criteria.

Example 1. Let us consider a bilinear time-varying delay system as follows: 


$$
\dot{x}(t)=A_{0} x(t)+A_{1} x(t-h(t))+N_{0} u(t) x(t)+N_{1} u(t-h(t)) x(t)+B u(t)
$$

where

$$
A_{0}=\left[\begin{array}{cc}
0 & 0 \\
0 & -2
\end{array}\right], A_{1}=\left[\begin{array}{ll}
2 & 0 \\
1 & 2
\end{array}\right], N_{0}=\left[\begin{array}{cc}
-0.3 & 0 \\
0 & -0.2
\end{array}\right], N_{1}=\left[\begin{array}{cc}
0.1 & 0 \\
0 & 0.2
\end{array}\right], B=\left[\begin{array}{l}
2 \\
0
\end{array}\right]
$$

Now, our problem is to design a memoryless state feedback controller as (5) to stabilize system (19) and estimate the bound of delay time $h$ to keep the stabililization of system.

Solution: Let $\rho=0.5, h_{d} \geq 1$, by using the LMI Toolbox in MATLAB (with accuracy 0.01), solving the following quasi-convex optimization problem (19). Then, the solutions of the LMI given in (17) are found to be $K=Y W^{-1}=[-56.8981-108.8760]$ and $h<12.5989$.

Example 2. Let us consider a bilinear time varying delay system as follows:

$$
\dot{x}(t)=A_{0} x(t)+A_{1} x(t-h(t))+N_{0} u(t) x(t)+N_{1} u(t-h(t)) x(t)+B u(t)
$$

where

$$
A_{0}=\left[\begin{array}{cc}
0 & 1 \\
0 & -5
\end{array}\right], A_{1}=\left[\begin{array}{ll}
3 & 0 \\
0 & 5
\end{array}\right], N_{0}=\left[\begin{array}{cc}
-0.3 & 0 \\
0 & -0.2
\end{array}\right], N_{1}=\left[\begin{array}{cc}
0.1 & 0 \\
0 & 0.2
\end{array}\right], B=\left[\begin{array}{c}
1 \\
0.5
\end{array}\right] .
$$

Now, our problem is to design a memoryless state feedback controller as (5) to stabilize system (20) and estimate the bound of delay time $h$ to keep the stabililization of system.

Solution: The results of the maximum allowable delay bound (MADB) $h$ for different values of $h_{d}$ are also listed in Table 1 . Note that the results of $[2,4,5,7,9,10]$ fail to deal with this system since the matrix describing the relationship between the slow and fast variables cannot be determined beforehand. For this case, it can be verified that stability conditions in $[3,11,12]$ do not apply when $h_{d} \geq 1$. Hence, for this example, the robust stability criterion of this paper is less conservative than the existing results [2, 3, 4, 5, 7, 9-12].

Table 1. Maximum allowable delay bound (MADB) $h$ for different $h_{d}(\rho=0.8)$

\begin{tabular}{|c|c|c|c|}
\hline$h_{d}$ & $h$ & Iterations & Feedback gains \\
\hline 0.5 & 500 & 21 & {$\left[\begin{array}{ll}-41.9844 & 41.2692\end{array}\right]$} \\
\hline 0.6 & 38.6899 & 15 & {$\left[\begin{array}{ll}-96.0227 & 120.9498\end{array}\right]$} \\
\hline 0.7 & 6.9297 & 16 & {$\left[\begin{array}{ll}-514.3448 & 799.8481\end{array}\right]$} \\
\hline 0.8 & 0.1847 & 28 & {$\left[\begin{array}{ll}-0.6771 & 1.0531\end{array}\right] \times 10^{7}$} \\
\hline 0.9 & 0.1655 & 17 & {$\left[\begin{array}{ll}-4.1691 & 5.4512\end{array}\right] \times 10^{4}$} \\
\hline$\geq 1$ & 0.1655 & 18 & {$\left[\begin{array}{ll}-0.7796 & 1.0193\end{array} \times 10^{5}\right.$} \\
\hline
\end{tabular}

\section{Conclusion}

In this paper, delay-dependent stability conditions and state feedback stabilization of bilinear systems with time-varying delays have been considered. The proposed design conditions have been casted in the form of LMIs feasibility problems, thus the control law can be efficiently computed by means of convex optimization algorithms. Finally, examples were given to illustrate our approach and to show the effectiveness over the existing results.

\section{REFERENCES}

[1] S. Boyd, L. El Ghaoui, E. Feron, V. Balakrishnan, Linear Matrix Inequalities in System and Control Theory, SIAM, Philadelphia, 1994.

[2] E. Fridman and U. Shaked, A descriptor system approach to $H_{\infty}$ control of linear time-delay systems, IEEE Transactions on Automatic Control, Vol. 47, pp. 253-270 2002. 
[3] Y. He, Q. G. Wang, L. H. Xie, and C. Lin. Further improvement of free-weighting matrices technique for systems with time-varying delay, IEEE Transactions on Automatic Control, Vol. 52, No.2, pp.293-299, 2007.

[4] P. L. Liu, and H. L. Hung, Stability criterion for bilinear systems with state-delay and saturating actuators, Proceeding of the 14th National Conference on Mechanical Engineering The Chinese Society of Mechanical Engineers, pp. 90-94, 1997.

[5] P. L. Liu, and H. L. Hung, Stability for bilinear time-delay systems with saturating actuators, Proceeding of the IEEE International Symposium on Industrial Electronics, pp. 1082-1086, 1999.

[6] P. L. Liu, A delay decomposition approach to stability analysis of uncertain systems with time-varying delays, ISA Transactions, Vol. 51, No.6, pp.694-701, 2012.

[7] H. C. Lu and C. A. Wey, Lyapunov stability of bilinear time-delay systems, Proceeding of 32nd IEEE Conference on Decision and Control, San Antonio, Texas, pp. 3841-3842, 1993.

[8] X. Y. Meng, J. Lam, B. Z. Du and H. J. Gao. A delay-partitioning approach to the stability analysis of discrete-time systems, Automatica, Vol.46, No.3, pp.610-614, 2010.

[9] T. J. Su, C. H. Liu and G. J. Jong, Robust stability for bilinear time-delay systems with saturating actuator, Proceeding of the IASTED International Conference Control and Applications, Banff, Canada, pp.70-74, 1999.

[10] J. Sun, G. P. Liu, J. Chen and D. Rees, Improved stability criteria for linear systems with time-varying delay. IET Control Theory Applications, Vol. 4, No.4, pp.683-689, 2010.

[11] S. H. Tsai and T.-H.S. Li, Robust fuzzy control of a class of fuzzy bilinear systems with time-delay, Chaos, Solitons and Fractals, Vol. 39, pp.2028-2040, 2009.

[12] J. Yoneyama, Output feedback control design for Takagi-Sugeno fuzzy bilinear time-delay systems, 2010 IEEE Conference on Systems, Man and Cybernetics, pp.1671-1677, 2010. 